\title{
Persistent Antibody Responses to SARS-CoV-2 Infection in Cancer Patients: A Single-Center Retrospective Observational Study
}

\author{
Amit Agarwal ${ }^{1}$ Saphalta Baghmar ${ }^{1}$ Suhail Qureshi ${ }^{1}$ Aseem Khurana ${ }^{1}$ Rasika Setia ${ }^{2}$ \\ Maroof Ahmad Khan ${ }^{3}$ Purabi Barman ${ }^{4}$ Raj Kumar Kapoor ${ }^{5}$ Navneeth Reddy Hasthavaram ${ }^{1}$ \\ Ramesh Kumar ${ }^{1}$ Navdeep Singh ${ }^{1}$ Aditya Sarin ${ }^{1}$ Anil Handoo ${ }^{6}$
}

${ }^{1}$ Department of Medical Oncology, Dr BL Kapur Super Specialty Hospital, New Delhi, India

${ }^{2}$ Department of Transfusion Medicine, Dr BL Kapur Super Specialty Hospital, New Delhi, India

${ }^{3}$ Department of Bio-Statistics, All India Institute of Medical Sciences (AlIMS), Ansari Nagar, New Delhi, India

${ }^{4}$ Department of Clinical Microbiology, Dr BL Kapur Super Specialty Hospital, New Delhi, India

\author{
Address for correspondence Amit Agarwal, DM, Department of \\ Medical Oncology, Dr BL Kapur Memorial Hospital, \\ New Delhi 110005, India (e-mail: deepaliamit@hotmail.com). \\ ${ }^{5}$ Department of Clinical Biochemistry, Dr BL Kapur Super Specialty \\ Hospital, New Delhi, India

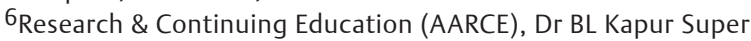 \\ Specialty Hospital, New Delhi, India
}

\begin{abstract}
Keywords

- COVID-19

- SARS-CoV-2

- anti-SARS-

CoV-2-antibody

- cancer

- seroconversion

- chemotherapy

- India

Introduction There is limited literature available regarding the prevalence and durability of immune response to infection with severe acute respiratory syndrome coronavirus 2 (SARS-CoV-2)/coronavirus disease 2019 (COVID-19) in cancer patients. Objective The aim of this study was to analyze the seroconversion rate in cancer patients recovered from SARS-CoV-2 infection.

Materials and Methods We retrospectively analyzed antibody levels and seroconversion rates in serum samples from 135 cancer patients who had recovered from SARS-CoV-2 infection. Chemiluminescent immunoassay using Roche Cobas e801 analyzer (Roche Diagnostics, Rotkreuz, Switzerland) was performed to identify Pan Ig antibody against nucleocapsid antigen. Reports of first, third, and sixth month were analyzed. Seroconversion was also compared with health-care workers (HCW) of our institute who had recovered from COVID-19 infection.

Results Seroconversion rate in cancer patients was $81.2 \%$ at 1 month, 95\% at 3 months, and $94.6 \%$ at 6 months post reverse transcriptase-polymerase chain reaction positivity. There was no difference in seroconversion rate among different age groups, gender, comorbidities, severity of COVID-19 symptoms, cancer disease status, and treatment with chemotherapy. Seroconversion rate in cancer patients is comparable to HCW (90.4 vs. $96 \%, p=0.82$ ) and is durable.

Conclusion Humoral response to COVID-19 infection in cancer patients is comparable to general population and sustained. Such responses suggest that cancer patients are likely to benefit from COVID-19 vaccination.
\end{abstract}

DOI https://doi.org/ $10.1055 / \mathrm{s}-0041-1733823$ ISSN 0971-5851 (c) 2021. Indian Society of Medical and Paediatric Oncology. This is an open access article published by Thieme under the terms of the Creative Commons Attribution-NonDerivative-NonCommercial-License, permitting copying and reproduction so long as the original work is given appropriate credit. Contents may not be used for commercial purposes, or adapted, remixed, transformed or built upon. (https://creativecommons.org/licenses/by-nc-nd/4.0/).

Thieme Medical and Scientific Publishers Private Ltd. A-12, Second Floor, Sector -2, NOIDA -201301, India 


\section{Introduction}

Severe acute respiratory syndrome coronavirus 2 (SARS-CoV-2), causing coronavirus disease 2019 (COVID-19), emerged in December 2019 and has spread around the world causing several cases and deaths. ${ }^{1}$ Patients suffering from cancer are thought to be at a higher risk of developing a severe course of COVID-19 and have higher mortality risk as their immune system is generally compromised. ${ }^{2,3}$ Due to this reason, adjuvant chemotherapies, surgeries, and other therapies are either postponed or altered during the current pandemic.

According to literature, seroconversion of most patients with COVID-19 occurs between 7 and 14 days after diagnosis. ${ }^{4-6}$ Earlier studies suggested that 40 to $60 \%$ of those infected become antibody-negative early in the convalescence period, ${ }^{7-10}$ while later studies reported sustained levels for at least 4 months $^{7,9,11,12}$ (Supplementary Table S1).

There is a paucity of data regarding SAR-CoV-2 seroconversion in cancer patients. ${ }^{13,14}$ (Supplementary Table S1) To the best of our knowledge, there is no data in literature that has studied longitudinal seroconversion of SAR-CoV-2 antibodies in this subset of patients. It is also not known if these antibodies have long-term persistence and whether they provide protective immunity against reinfection in cancer patients.

Response to pneumococcal and influenza vaccination has shown good response in patients on chemotherapy. ${ }^{15,16}$ However, COVID-19 vaccine trials have excluded cancer/ immunocompromised patients. ${ }^{17-19}$ Hence, there is no data on the safety and efficacy of COVID-19 vaccine in cancer patients.

The aim of this study was to assess SARS-CoV-2 seroconversion in the cancer patients and longitudinal changes of antibody levels in first 6 months after SARS$\mathrm{CoV}-2$ infection. We also assessed its correlation with sex, age, anticancer treatment, and severity of SARS-CoV-2 infection. Comparison was done with seroconversion in normal population comprising of 100 recovered frontline health-care workers (HCW) with confirmed COVID-19 infection.

\section{Materials and Methods}

\section{Study Design}

This study was a retrospective analysis evaluating serial estimation of anti-SARS-CoV2-antibody in cancer patients who recovered from SARS-CoV2 infection.

\section{Study Setting}

Study was performed at a tertiary care hospital, The study period was from April 30 to December 25, 2020.

\section{Data Collection}

All the demographic data, clinical information, laboratory parameters, and complications during the hospital stay were retrieved from hospital medical records. Cases of acute leukemia were not included in the study. All the data was analyzed at the cutoff date of December 25, 2020.

\section{Inclusion Criteria}

All cancer patients who tested positive for SARS-CoV-2 by reverse transcriptase-quantitative polymerase chain reaction (RT-qPCR) on nasal/throat swab and were tested at least once for SARS-CoV2-antibodies were enrolled in this retrospective analysis.

\section{Exclusion Criteria}

1. All leukemia patients.

2. All cancer patients below age 18 years.

\section{Primary Outcome}

Seroconversion rate in cancer patients recovered from SARS-CoV-2 infection at first, third, and sixth month.

\section{Secondary Outcome}

Comparison of seroconversion rate among cancer patients and healthy HCWs (internal control).

\section{Diagnostic Criteria}

SARS-CoV-2 infection: The severity of the disease was defined according to the World Health Organization (WHO) definition..$^{20}$

\section{Detection of SARS-CoV-2 RNA by RT-PCR}

Presence of SARS-CoV-2 on nasopharyngeal/throat swab specimens was determined by real-time RT-PCR. Allplex 2019 n-CoV Assay (Seegene Inc., Seoul, South Korea) was used to detect SARS-CoV-2 by amplification of RdRp gene, $\mathrm{E}$ gene, and $\mathrm{N}$ gene according to the Indian Council of Medical Research (ICMR) recommendations. ${ }^{21}$

\section{Detection of SARS-CoV-2 Antibodies}

We tested our patient samples using Roche Elecsys anti-SARS-CoV-2 immunoassay (Roche Diagnostics, Rotkreuz, Switzerland) on a Cobas e801 analyzer. Elecsys anti-SARS-CoV-2 electrochemiluminescence immunoassay detects antibodies (including immunoglobulin M [IgM], immunoglobulin A [IgA], and immunoglobulin $G$ [ $\operatorname{IgG}]$ ) to SARS-CoV-2 in human serum. The immunoassay utilizes a double-antigen sandwich test principle and a recombinant protein representing the nucleocapsid antigen for the determination of antibodies to SARS-CoV-2. Tests were performed according to the manufacturer's instructions, and assay results were interpreted as follows: cutoff index $\geq 1.0$ was considered as positive/reactive. ${ }^{22}$

In a previous study done at our institute (COVID-19 recovered HCW cohort), ${ }^{23}$ we compared the performance of four high-through put commercial chemiluminescence immunoassays [Abbott Architect SARS-CoV-2 IgG assay which detects antinucleocapsid IgG, Roche Elecsys anti-SARS-CoV-2 total assay antinucleocapsid (IgG, IgM \& IgG), Ortho-Clinical Diagnostics VITROS anti-SARS-CoV-2 IgG (S1) and OrthoClinical Diagnostics VITROS anti-SARS-CoV-2 total antispike 
\{including IgA, IgM \& IgG (S1)\} to SARS-CoV-2] most frequently used for the detection of SARS-CoV-2 antibodies worldwide. Comparisons were done in terms of various statistical parameters like sensitivity, specificity, and Cohen's kappa agreement (- Fig. 1).

Results from antinucleocapsid antibody ( $\operatorname{IgG}$, IgM, and $\operatorname{Ig} \mathrm{A}$ ) on Roche platform (used subsequently for our study) were in complete agreement with IgG (S1) and total antispike done on Ortho-Clinical Diagnostics platform (Supplementary Tables S2 and S3).

For the purpose of analysis, we divided first month data in three subsets from the day of COVID-19 RT-PCR positivity, that is, less than 15 days, 15 to 22 days, 23 to 30 days, then at 3 and 6 months.

\section{Sample Size}

All consecutive cancer patients fulfilling the inclusion criteria during the study period were included. A total of 135 patients were included in the study.

\section{Statistical Analysis}

To describe patient's characteristic's, demographic, clinical, laboratory investigations, the data were summarized and analyzed using STATA (version 14, StataCorp., College Station, Texas, United States) software. Quantitative data was expressed as median ( $\min , \max$ ). Qualitative data was reported as in numbers and percentages. Data was tested for normality using the Kolmogorov-Smirnov test. Student's $t$-test was used to observe the differences between demographic factors, cancer history, clinical findings, laboratory parameters and disease outcome, between patients who developed anti-SARS-CoV2-antibodies after COVID-19 infections versus those who did not. Comparison of baseline and treatment characteristics was performed using Student's t-test. To establish the association between patients who developed antibodies versus those who did not, with other qualitative characteristics, chi-squared test/Fisher's exact test was used. A value of $p$ less than 0.05 was considered to represent statistical significance of the study.

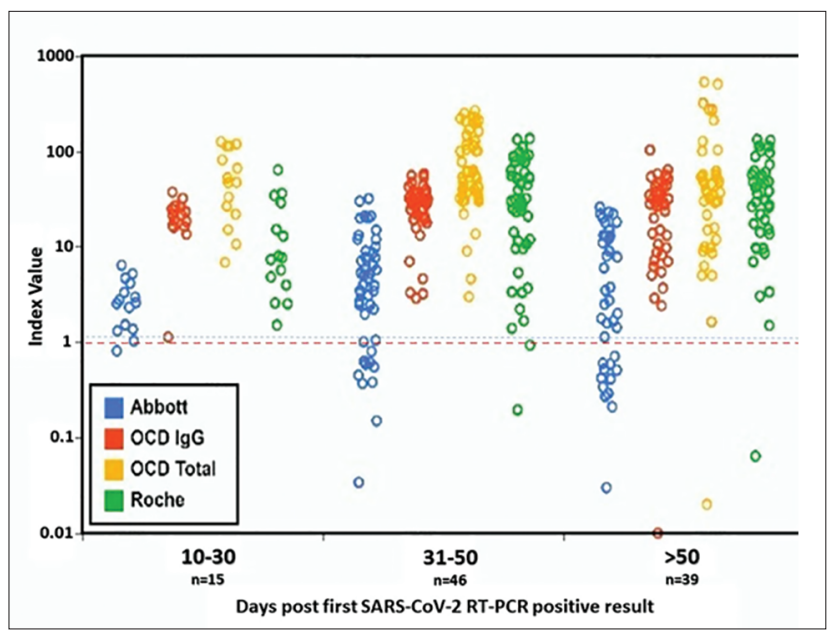

Fig. 1 Comparison of chemiluminescent immunoassay platforms.
Ethics

The study protocol was reviewed and approved by institute ethics committee (Dr. BL Kapur Memorial Hospital Ethics Committee) on January 7, 2021. The approval letter number is ETHICS COMMITTEE/AARCE/LETTER/JAN/2021/12.

Consent waiver was granted given retrospective nature of analysis confidentiality was maintained by the deidentification of data. The procedures followed were in accordance with the ethical standards of the responsible committee on human experimentation and with the Helsinki Declaration of 1964 , as revised in 2013.

\section{Results}

\section{Patient Characteristics and Disease Status}

A total of 135 patients were enrolled in the study. Their median age was 56 years (range: $24-80$ years) and median follow-up was 92 days (range: 12-239).

Details of site of tumor, stage, disease status, and preceding cancer treatment before COVID-19 infection are shown in - Table 1. Overall, 26 (19\%) patients were classified with moderate or severe symptoms, and 41 (30\%) patients were asymptomatic. Median duration of symptoms was 2 days (range: 0-10).

\section{COVID-19 Antibody}

Seroconversion rate of overall population was 90.4\% (122 of 135). This was $75 \%$ at 15 days, $78 \%$ at 15 to 22 days, and $84 \%$ at 23 to 30 days. It increased to $95 \%$ at third month and maintained at $94.6 \%$ at sixth month (- Table 2 ).

Multiple clinical and laboratory covariates were assessed for association with antibody production (age, sex, stage, type of cancer, COVID-19 severity, etc.) and none were found to be correlating with antibody presence. Notably, type, and treatment of cancer and severity of COVID-19 symptoms did not affect production of antibody and its durability (-Table $\mathbf{1}$ ).

\section{Seroconversion in Cancer Patients as Compared with HCWs}

A total of 96 of 100 HCWs had antinucleocapsid antibodies positivity. Overall seroconversion rate in cancer patients was $90.4 \%$ that was comparable with $\mathrm{HCW}(96 \% ; p=0.82)$.

\section{Loss of Seroconversion}

Only three $(2.4 \%)$ patients became seronegative in subsequent evaluation. All three had borderline positivity for antibody (1.3-2.88 index value) and loss happened at 33, 112, and 127 days, respectively.

\section{Discussion}

Studies evaluating seroconversion in COVID-19 infected individuals have shown results approaching 90\%-12 (Supplementary Table S1). There are studies showing its subsequent loss, ${ }^{7-10}$ whereas a large study from Iceland 
Table 1 Baseline demographics and cancer therapy details

\begin{tabular}{|c|c|c|c|c|}
\hline Variable & $n(\%)$ & $\begin{array}{l}\text { COVID-19 antibody } \\
\text { positive patients }\end{array}$ & $\begin{array}{l}\text { COVID-19 antibody } \\
\text { negative patients }\end{array}$ & $p$-Value \\
\hline \multicolumn{5}{|l|}{ Age } \\
\hline Median (range) & $\begin{array}{l}56 \\
(24-80)\end{array}$ & & & \\
\hline$\leq 60 y$ & $93(61)$ & 85 & 8 & \\
\hline$>60 y$ & $42(39)$ & 37 & 5 & 0.376 \\
\hline \multicolumn{5}{|l|}{ Sex } \\
\hline Male & $39(29)$ & 35 & 4 & \\
\hline Female & $96(71)$ & 87 & 9 & 0.55 \\
\hline \multicolumn{5}{|l|}{ Type of tumor } \\
\hline Breast & $50(37)$ & 46 & 4 & \\
\hline Lung & $8(5.7)$ & 5 & 3 & \\
\hline Head and neck & $5(3.7)$ & 4 & 1 & \\
\hline Hepato-Pancreato- & $11(7.4)$ & 11 & 0 & \\
\hline Biliary & $20(15.5)$ & 18 & 2 & \\
\hline Gastrointestinal & $8(5.7)$ & 8 & 0 & \\
\hline Genitourinary & $14(10.3)$ & 14 & 0 & \\
\hline Gynecological & $8(5.7)$ & 8 & 0 & \\
\hline \multicolumn{5}{|l|}{ Others } \\
\hline \multicolumn{5}{|l|}{ Hematological tumors } \\
\hline Lymphoma & $8(5.7)$ & 5 & 3 & 0.03 \\
\hline Multiple myeloma & $3(2.2)$ & 3 & 0 & \\
\hline \multicolumn{5}{|l|}{ Cancer status } \\
\hline Cured + remission & $40(29.6)$ & 36 & 4 & \\
\hline $\begin{array}{l}\text { Response to active disease or treatment naïve } \\
\text { or relapse/refractory disease }\end{array}$ & $95(70.4)$ & 86 & 9 & 0.57 \\
\hline \multicolumn{5}{|l|}{ Comorbidity } \\
\hline None & $81(60)$ & 74 & 7 & \\
\hline One & $29(21.5)$ & 25 & 4 & \\
\hline$\geq$ two & $25(18.5)$ & 23 & 2 & 0.71 \\
\hline \multicolumn{5}{|l|}{ Cancer stage } \\
\hline I-III & $55(40.7)$ & 49 & 6 & \\
\hline IV & $80(59.3)$ & 73 & 7 & 0.77 \\
\hline \multicolumn{5}{|l|}{$\begin{array}{l}\text { Chemotherapy (received) within last } 4 \\
\text { weeks }(n=98)\end{array}$} \\
\hline Cytotoxic & 67 (49.6) & 60 & 7 & \\
\hline Targeted & $9(6.7)$ & 9 & 0 & \\
\hline Hormonal & $18(13.3)$ & 17 & 1 & \\
\hline Immunotherapy & $5(3.7)$ & 5 & 0 & 0.86 \\
\hline \multicolumn{5}{|l|}{ Symptoms } \\
\hline Fever & $76(56.3)$ & 70 & 6 & 0.31 \\
\hline Cough & $55(40.7)$ & 50 & 5 & 0.55 \\
\hline
\end{tabular}


Table 1 (continued)

\begin{tabular}{|c|c|c|c|c|}
\hline Variable & $n(\%)$ & $\begin{array}{l}\text { COVID-19 antibody } \\
\text { positive patients }\end{array}$ & $\begin{array}{l}\text { COVID-19 antibody } \\
\text { negative patients }\end{array}$ & $p$-Value \\
\hline Sore throat & $46(34)$ & 43 & 3 & 0.29 \\
\hline Myalgia & $75(55.6)$ & 70 & 5 & 0.15 \\
\hline Anosmia & $34(25.2)$ & 33 & 1 & 0.11 \\
\hline Dysguesia & $36(26.7)$ & 35 & 1 & 0.09 \\
\hline \multicolumn{5}{|l|}{ Symptoms duration (d) } \\
\hline Median & 2 & 71 & 7 & \\
\hline$\leq 2 \mathrm{~d}$ & $78(57.8)$ & 51 & 6 & 0.59 \\
\hline$>2 d$ & $57(42.2)$ & & & \\
\hline \multicolumn{5}{|l|}{ Severity } \\
\hline Asymptomatic + mild & $\begin{array}{l}109 \\
(80.7)\end{array}$ & 98 & 11 & \\
\hline Moderate + severe & $26(19.3)$ & 24 & 2 & 0.52 \\
\hline Survivors & $123(91)$ & 113 & 10 & \\
\hline Nonsurvivors & $12(9)$ & 9 & 3 & 0.09 \\
\hline
\end{tabular}

Abbreviation: COVID-19, coronavirus disease 2019.

Table 2 COVID-19 antibody positivity

\begin{tabular}{|c|l|}
\hline $\begin{array}{l}\text { COVID-19 antibody test } \\
\text { time (d) }\end{array}$ & Positive test/test done (\%) \\
\hline$<1-30$ & $65 / 80(81.25)$ \\
\hline$<15 d$ & $9 / 12(75)$ \\
\hline $15-22$ & $25 / 32(78)$ \\
\hline $23-30$ & $32 / 38(84)$ \\
\hline $61-90$ & $39 / 41(95)$ \\
\hline $151-180$ & $35 / 37(94.6)$ \\
\hline
\end{tabular}

Abbreviation: COVID-19, coronavirus disease 2019.

showed persistence of antibodies for at least 4 months in $\geq 90 \%$ of recovered patients ${ }^{11}$ (Supplementary Table S1). Persistence of antibodies has also been documented by other studies. ${ }^{9,12}$

\section{Anti-COVID-19 Antibody in Cancer Patients}

Seroconversion rate in our study population was $81.25 \%$ by first month, $95 \%$ at third month, and $94 \%$ by six month.

There are two published studies addressing positivity of antibody in cancer patients with COVID-19 infection. Solodky et al reported 30\% antibody positivity rate (3 out of 10 patients) in cancer patients at day 15 or later after clinical symptoms as compared with $71 \%$ in noncancer patients (30 vs. $71 \%, p=0.04$ ). ${ }^{13}$ However, this was a small study with only 10 cancer patients. A study from Wuhan showed anti-COVID-19 antibody prevalence of $72.5 \%$ in 40 cancer patients (Supplementary Table S1). ${ }^{14}$ In this study, the antibodies were tested at 21 days from symptom onset, whereas our study calculated time from RT-PCR positivity (often performed after appearance of symptoms). Our 15 days' data of $75 \%$ seroconversion is comparable to this study. Our larger study reinforces early appearance of antibodies in majority of cancer patients.

In our study cohort, overall, 90.4\% seropositivity was seen at the median follow-up of 92 days, which was similar to general population reported in previous studies. ${ }^{10,11}$ We also compared overall seroconversion rate of cancer patients with HCW at our institute and found it similar (90.4 vs. 96\%). Patients suffering from cancer have equivalent humoral responses compared with general population.

In our study, 35 out of 37 patients showed persistence of COVID-19 antibodies at sixth month. No loss of antibodies was observed between third and sixth month. Persistence of antibodies up to 6 months has been shown for the first time in cancer patients.

In the study, 67 patients received chemotherapy within 4 weeks prior to COVID-19 infection. Seroconversion rate among these patients was $90 \%$ (at a median of 33 days) that is similar to the overall cohort of cancer patients. We did not observe any poor antibody responses in patients on chemotherapy.

Protective nature of these antibodies has been documented by studies on pseudoneutralization assay ${ }^{24-26}$ and clinical data on reinfection rates. ${ }^{27,28}$ Though the titer of antibody that can be considered to be protective is not known, maintained antibody levels are reassuring. Low incidence of reinfection worldwide is another indirect evidence of its protective nature. Sustained responses can possibly prevent reinfection in cancer patients thus avoiding treatment delays, morbidity, and mortality. None of our patients had 
reinfection till the date of censoring. This is the first such study to report this in patients suffering from cancer.

\section{Low Levels May Become Negative}

Of 135 patients, 10 patients had antibody levels between 1 and 3 index values at time of first test. Of these 10 , three patients (30\%) lost their antibody levels in subsequent testing. The patients with low index value need serial testing. Such cases may represent false positive or lower immune responses. These are small numbers and more studies are required to assess true incidence and implication of this subset.

\section{Rituximab}

In the cohort, eight patients were of non-Hodgkin lymphoma. Six out of these eight patients had received rituximab in the past. Among these six patients, three patients did not seroconvert and the other three patients had very low titer of antibodies. Rituximab may thus be playing a role in blunted humoral responses. ${ }^{29}$ Lower immune responses possibly can increase the risk of reinfection and also can impact response to vaccination.

\section{Vaccination in Cancer Patients}

Pfizer and AstraZeneca trials have excluded patients of cancer/immunosuppressed states from vaccine trial, ${ }^{17,18}$ whereas Moderna ${ }^{19}$ does not have clear information regarding the same. Hence, we do not know how the cancer patients would develop immune response to the same. Our data so far supports for the vaccination of cancer patients as there is no adverse impact on antibody production in them even on chemotherapy. The same cannot be said regarding patients on rituximab.

Limitations of the study are its retrospective nature and unadjusted demographic data. Reference date for calculation was taken as date of COVID-19 RT-PCR positivity but patients had symptoms before that. So, the date of antibody positivity may not be the actual date from COVID-19 infection. This data is most mature for 3 months as the median follow-up is 92 days and inference on stability of antibody at 6 months is based on a small number of patients (27.4\%).

\section{Future Research Directions}

The study is ongoing and we would come up with 9 months follow-up. Longer follow-up will provide the information regarding the durability of COVID-19 antibody response in cancer patients.

\section{Conclusion}

To conclude, cancer patients even on chemotherapy produce sustained and equivalent antibody response to COVID-19 as general population. Their reinfection rates are low. Cancer patients are therefore likely to respond well to COVID-19 vaccine and should be considered as priority group due to known high case fatality. However, antibody response in rituximab treated patients may be suboptimal and needs further assessment.

\section{Disclaimers}

The views expressed in the submitted article are my own and not an official position of the institution or funder.

The manuscript has been read and approved by all the authors, that the requirements for authorship as stated in the instruction manual have been met, and that each author believes that the manuscript represents honest work.

\section{Funding \\ Not applicable.}

\section{Conflict of Interests}

No financial and personal conflict of interest.

\section{References}

1 COVID-19. Coronavirus Pandemic. https://www.worldometers info/coronavirus/. Accessed July 12, 2021

2 Liang W, Guan W, Chen R, et al. Cancer patients in SARSCoV-2 infection: a nationwide analysis in China. Lancet Oncol 2020;21(3):335-337

3 Onder G, Rezza G, Brusaferro S. Case-fatality rate and characteristics of patients dying in relation to COVID-19 in Italy. JAMA 2020;323(18):1775-1776

4 Vabret N, Britton GJ, Gruber C, et al. Sinai Immunology Review Project. Immunology of COVID-19: current state of the science. Immunity 2020;52(6):910-941

5 Zhao J, Yuan Q, Wang H, et al. Antibody responses to SARS$\mathrm{CoV}-2$ in patients of novel coronavirus disease 2019. Clin Infect Dis 2020;71(16):2027-2034

6 Pollán M, Pérez-Gómez B, Pastor-Barriuso R, et al. ENE-COVID Study Group. Prevalence of SARS-CoV-2 in Spain (ENE-COVID): a nationwide, population-based seroepidemiological study. Lancet 2020;396(10250):535-544

7 Long Q-X, Tang X-J, Shi Q-L, et al. Clinical and immunological assessment of asymptomatic SARS-CoV-2 infections. Nat Med 2020;26(8):1200-1204

8 Ward H, Cooke GS, Atchison C, et al. Prevalence of antibody positivity to SARS-CoV-2 following the first peak of infection in England: Serial cross-sectional studies of 365,000 adults. The Lancet Regional Health - Europe, 2021;4:100098. ISSN 2666-7762. Available at: https://doi.org/10.1016/j. lanepe.2021.100098

9 Bölke E, Matuschek C, Fischer JC. Loss of anti-SARS-CoV-2 antibodies in mild Covid-19. NEnglJ Med 2020;383(17):1694-1695

10 Ibarrondo FJ, Fulcher JA, Goodman-Meza D, et al. Rapid decay of anti-SARS-CoV-2 antibodies in persons with mild Covid-19. N Engl J Med 2020;383(11):1085-1087

11 Gudbjartsson DF, Norddahl GL, Melsted P, et al. Humoral immune response to SARS-CoV-2 in Iceland. N Engl J Med 2020;383(18):1724-1734

12 prpt . Wajnberg A, Amanat F, Firpo A, et al. SARS-CoV-2 infection induces robust, neutralizing antibody responses that are stable for at least three months. July 17, 2020 ( https://www. medrxiv. org/ content/ 10.1101/ 2020. 07. 14. 20151126v1)./ prpt

13 Solodky ML, Galvez C, Russias B, et al. Lower detection rates of SARS-COV1 antibodies in cancer patients vs healthcare workers after symptomatic COVID-19. Annals of Oncology. doi:10.1016/j.annonc.2020.04.475.\} DOI: https://doi. org/10.21203/rs.3.rs71560/v1\}/prpt

14 Liu T, Zeng G, Tao H, et al. COVID-19 in Cancer Patients Research Group. Low prevalence of IgG antibodies to SARSCoV-2 in cancer patients with COVID-19. Int J Cancer 2020;147(11):3267-3269 
15 Berglund A, Willén L, Grödeberg L, Skattum L, Hagberg $\mathrm{H}$, Pauksens $\mathrm{K}$. The response to vaccination against influenza $A(H 1 N 1)$ 2009, seasonal influenza and Streptococcus pneumoniae in adult outpatients with ongoing treatment for cancer with and without rituximab. Acta Oncol 2014;53(9):1212-1220

16 Nordøy T, Aaberge IS, Husebekk A, et al. Cancer patients undergoing chemotherapy show adequate serological response to vaccinations against influenza virus and Streptococcus pneumoniae. Med Oncol 2002;19(2):71-78

17 Polack FP, Thomas SJ, Kitchin N, et al. C4591001 Clinical Trial Group. Safety and efficacy of the BNT162b2 mRNA Covid-19 vaccine. N Engl J Med 2020;383(27):2603-2615

18 Folegatti PM, Ewer KJ, Aley PK, et al; Oxford COVID Vaccine Trial Group. Safety and immunogenicity of the ChAdOx1 $\mathrm{nCoV}-19$ vaccine against SARS-CoV-2: a preliminary report of a phase $1 / 2$, single-blind, randomised controlled trial. Lancet 2020;396(10249):467-478

19 Jackson LA, Anderson EJ, Rouphael NG, et al. mRNA-1273 Study Group. An mRNA vaccine against SARS-CoV-2 - preliminary Report. N Engl J Med 2020;383(20):1920-1931

20 World Health Organization. Clinical management of COVID-19: interim guidance. May 27, 2020.

21 https://www.icmr.gov.in/pdf/covid/labs/2_SOP_for_ Confirmatory_Assay_for_2019_nCoV.pdf. Accessed July 12, 2021

22 Roche. Elecsys ${ }^{\circledR}$ Anti-SARS-CoV-2. Package Insert 2020-07, V3.0; Material Numbers 09203095190 and 09203079190. US Food and Drug Administration https://www.fda.gov/ media/137605/download. Accessed July 12, 2021
23 Setia R, Dogra M, Handoo A, et al. Performance Evaluation: Four Chemiluminescent SARS-Cov-2 Immunoassays and Rapid-Card Test in Mild Disease and Seroprevalence of SARS CoV-2 in Frontline Healthcare Workers. Int Blood Res Rev 2021;12(3):9-22. Available at: https://doi.org/10.9734/ ibrr/2021/v12i330151

24 Muench P, Jochum S, Wenderoth V, et al. Development and validation of the Elecsys Anti-SARS-CoV-2 immunoassay as a highly specific tool for determining past exposure to SARSCoV-2. J Clin Microbiol 2020;58(10):e01694-e20

25 Nie J, Li Q, Wu J, et al. Establishment and validation of a pseudovirus neutralization assay for SARS-CoV-2. Emerg Microbes Infect 2020;9(1):680-686

26 Lei C, Qian K, Li T, et al. Neutralization of SARS-CoV-2 spike pseudotyped virus by recombinant ACE2-Ig. Nat Commun 2020;11(1):2070

27 Lumley SF, O’Donnell D, Stoesser NE, et al. Oxford University Hospitals Staff Testing Group. Antibody status and incidence of SARS-CoV-2 infection in health care workers. N Engl J Med 2021;384(6):533-54010.1056/NEJMoa2034545

28 prpt. Harvey RA, Rassen JA, Kabelac CA, et al. Real-world data suggest antibody positivity to SARS-CoV-2 is associated with a decreased risk of future infection. medRxiv 2020; https:// orcid.org/0000-0001-9372-9869/prpt

29 Houot R, Levy R, Cartron G, Armand P. Could anti-CD20 therapy jeopardise the efficacy of a SARS-CoV-2 vaccine? Eur J Cancer 2020;136:4-6 